\title{
Distinct Organizational States of Fully Developed Turbulent Pipe Flow
}

\author{
David J. C. Dennis ${ }^{*}$ and Francesca M. Sogaro \\ School of Engineering, University of Liverpool, Liverpool L69 3GH, United Kingdom
}

(Received 21 March 2014; published 3 December 2014)

\begin{abstract}
Organizational states of turbulence are identified through novel analysis of large scale pipe flow experiments at a Reynolds number of 35000 . The distinct states are revealed by an azimuthal decomposition of the two-point spatial correlation of the streamwise velocity fluctuation. States with dominant azimuthal wave numbers corresponding to $k_{\theta}=2,3,4,5,6$ are discovered and their structure revealed as a series of alternately rotating quasistreamwise vortices. Such organizational states are highly reminiscent of the nonlinear traveling wave solutions previously identified at Reynolds numbers an order of magnitude lower.
\end{abstract}

DOI: 10.1103/PhysRevLett.113.234501

PACS numbers: 47.27.N-, 47.27.De, 47.60.-i

There are two very distinct approaches to turbulence research. One is based on a dynamical systems viewpoint and the approach to the problem is to try to capture its essence in a mechanistic, low-dimensional system. Importantly, this approach has so far only been effective at very low Reynolds numbers (Re) close to transition. The other approach is a statistical one that has been employed for many years (mostly using experimental data) to study turbulent flows at a wide range of Reynolds numbers (even very high $\mathrm{Re}$ ). In this Letter we show that the dynamical systems picture of coherent structures organizing the dynamics close to transition persists at higher Reynolds numbers. We identify the corresponding structures and show that they resemble their low-Re counterparts.

Using the dynamical systems approach, significant advances in turbulence research have been made through applying ideas from finite-dimensional dynamical systems theory to the governing equations of fluid flow (the NavierStokes equations) [1]. This has led to the discovery of "exact coherent states" [2,3], which are essentially invariant solutions of the Navier-Stokes equations, in turbulent flow. A prime example is that of unstable, nonlinear traveling wave solutions in a turbulent pipe flow close to transition [4-9].

In recent years, the more traditional approach has also made great progress through increasingly sophisticated experimental methods [10] and direct numerical simulations (DNS) of turbulence [11] with regard to the presence of coherent motions in turbulent flows (of which fully developed turbulent pipe flow is one example [12]). The discovery and subsequent characterization of several types of coherent motions, such as bursts, sweeps, vortices, and

Published by the American Physical Society under the terms of the Creative Commons Attribution 3.0 License. Further distribution of this work must maintain attribution to the author(s) and the published article's title, journal citation, and DOI. vortex packets or clusters [13-19], and very large-scale motions (VLSMs) [20-24], has provided a new perspective in which to view turbulent flow and has hinted at a potentially decipherable organization to the flow.

The two approaches have both developed an idea of turbulence as consisting of a jungle of coherent structures or coherent motions. Despite this, the two approaches still remain quite separate. The biggest difficulty in bridging this divide is the Reynolds number. Whereas experimentalists are often primarily concerned with investigating turbulent behavior at increasingly high Re, dynamical systems theory is necessarily applied to low Re flows. This difficulty can lead one to question the relevance of the low Re exact coherent states unless they can be identified in high Re turbulent flows, which are evidently more complex than any hitherto identified invariant solution. Despite only being applied at low Re, the dynamical systems viewpoint justifies its relevance to higher Re flow on the basis that there is nothing particularly special about Reynolds numbers near transition and the same mechanistic picture can be applied at any Reynolds number, except that as the Reynolds number increases the number of exact solutions also increases. However, nobody has ever been able to prove this assertion and the present evidence base is not particularly strong. What is required is experimental evidence to bring the two approaches together at an intermediate Reynolds number well above transition.

The question of how experimental data can best be analyzed in order to show the presence (or otherwise) of exact coherent states is an open one. In this Letter we present, through the use of a novel analysis technique applied to a large-scale experiment, evidence suggestive of the presence of nonlinear traveling-wave invariant solutions in fully developed turbulent pipe flow at a Reynolds number significantly higher than that at which they have been previously observed. In so doing we not only provide the first evidence for the potential relevance of dynamical systems based solutions applied to higher Re flows, but 
also illuminate new ways in which turbulence data can be analyzed with a view to linking these two (often far too separate) fields of research.

The experiments were performed in the Very Large Scale Pipe Flow (VLSPF) facility at the University of Liverpool. This facility consists of a $23.3 \mathrm{~m}$ long pipe constructed of a series of borosilicate glass sections with an internal diameter $100 \mathrm{~mm}$. The turbulent flow at $\operatorname{Re}=U_{b} D / \nu=35000$ and $R^{+}=u_{\tau} R / \nu=940$, (where $U_{b}=$ bulk velocity, $D=$ pipe diameter, $R=$ pipe radius, $u_{\tau}=$ friction the velocity, and $\nu=$ kinematic viscosity) is investigated using a high-speed, stereoscopic particle image velocimetry technique $[18,23,25]$ in which the measurement plane (located $22 \mathrm{~m}$ from the pipe inlet, corresponding to $220 \mathrm{D}$ ) is perpendicular to the streamwise velocity, providing all three components of velocity across the entire pipe cross section with good temporal resolution. The flow is not tripped but transitions to turbulence naturally (at a Reynolds number of approximately 2000) a little way downstream of the inlet. Immediately upstream of the inlet is a plenum chamber to remove swirl and help provide uniform inlet flow. To enable the PIV measurements the flow is seeded with silver-coated hollow glass spheres with an average diameter of $10 \mu \mathrm{m}$, which have a density close to that of water and hence follow the turbulent flow closely. A water-filled prism is located between the pipe and cameras to eliminate refraction caused by the air-glass interface. The results presented in this Letter come from 21268 vector fields, or the equivalent of $512.4 R$ of fluid passing the measurement plane.

The allocation of each individual velocity field to a specific state (i.e., a single wave number) was based on the number of positive-negative peaks present in the instantaneous streamwise velocity fluctuation of that field alone. First, the 2D spatial correlation was calculated using Eq. (1), where the zero subscripts denote the center of the correlation. Because of the azimuthal symmetry, the correlation can be performed through all azimuthal angles and ensemble averaged. The azimuthal wave number of the resulting correlation was identified using the highest peak in fast Fourier transform (FFT). Over $97 \%$ of the vector fields could be assigned to a wave number between 2 and 6 using this technique. Wave numbers of 1 and 7 were also detected but they were very rare and didn't provide converged statistics, so they have not been presented. No threshold was used on the peak height. The application of thresholds was investigated but using a threshold to match only the most coherent instances (similar to [26]) resulted in only marginal differences in the results despite only $25 \%$ of the vector fields being assigned to a state. We have therefore resisted the temptation to use an arbitrary threshold.

$R_{u u}\left(r_{0}+\Delta r, \theta_{0}+\Delta \theta\right)=\frac{\left\langle u\left(r_{0}+\Delta r, \theta_{0}+\Delta \theta\right) u\left(r_{0}, \theta_{0}\right)\right\rangle}{u_{\mathrm{rms}}^{2}}$.
Figure 1(a) shows the two-point spatial correlation of the streamwise velocity fluctuation in the radial-azimuthal plane decomposed by azimuthal wave number. We show the five dominant wave numbers $\left(k_{\theta}=2,3,4,5,6\right)$. A clear alternating pattern of positive and negative correlation is observable at each of the wave numbers. These are related to the large-scale coherent motions in the turbulent flow and elucidate a behavior that involves significant correlation at large azimuthal angles. This is demonstrated in Fig. 1(b), which shows an example instantaneous velocity field for each of the five wave number states. The alternating pattern is observable in the streamwise velocity fluctuations, although it is to some extent camouflaged by the small-scale fluctuations, particularly at $k_{\theta}=6$.

Figures 1(c) and 1(d) show the conditionally averaged velocity field for each wave number state. The color map shows streamwise velocity fluctuation and the vector arrows indicate the direction of the flow in the azimuthal-radial plane. In addition to applying the condition corresponding to the wave number, a condition on the sign of the streamwise velocity fluctuation has also been enforced. Figure 1(c) is conditioned for $u_{0}<0$ and Fig. 1(d) for $u_{0}>0$, where $u_{0}=u\left(r_{0}, \theta_{0}\right)$. All of the assignable vector fields are used and the split between $u_{0}<0$ and $u_{0}>0$ is approximately even for all wave numbers $(50 \% \pm 2 \%)$. The vector arrows have been normalized such that their magnitudes are all equal for the purposes of clearly showing the vortices around the whole azimuth of the pipe; without the normalization, the vortices around the azimuth are swamped by the vectors near the conditioning point. These figures encapsulate the characteristics of each of the states: alternating positive and negative fluctuations of the streamwise velocity around the pipe azimuth combined with a series of alternately rotating quasistreamwise vortices. It is interesting to observe the variation in radial location of the vortex cores around the pipe azimuth. They are not all located at the height at which the condition is applied, instead a wide variation can be observed, with some located near the wall and others located further towards the center of the pipe. This is particularly noticeable for $k_{\theta}=4$ and $k_{\theta}=5$ in our experimental results. This variation is due to the conditioning on $u_{0}$. Physically, it can be interpreted as the flow pattern centered around a low-speed $\left(u_{0}<0\right)$ event being different to that centered around a high speed event $\left(u_{0}>0\right)$ even within the same flow field. This is a distinct characteristic of some previously discovered travelingwave solutions in turbulent pipe flow and the overall picture is very reminiscent of a set of nonlinear traveling wave solutions $[4,5]$. The only previous experimental observation of such states is from instantaneous snapshots at much lower Re [27,28]. It is worth noting that the "edge state" identified in numerical simulations at Reynolds numbers near transition [29] would be classified as a $k_{\theta}=$ 2 state in our system and, in fact, the conditional average of 

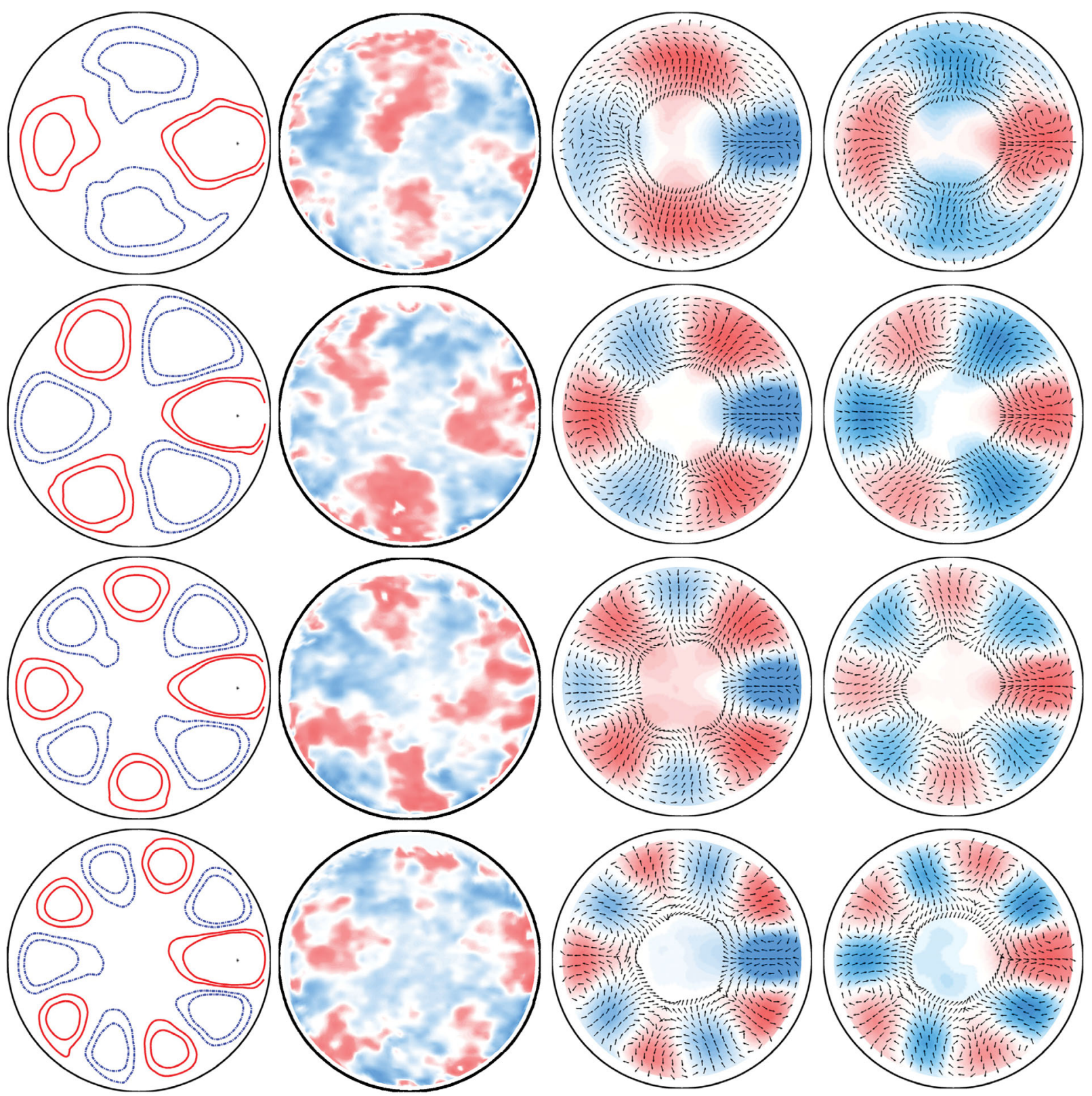

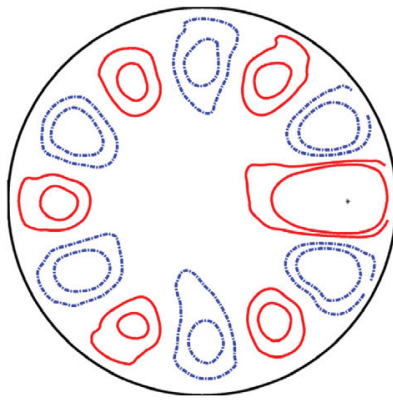

(a) Spatial correlation

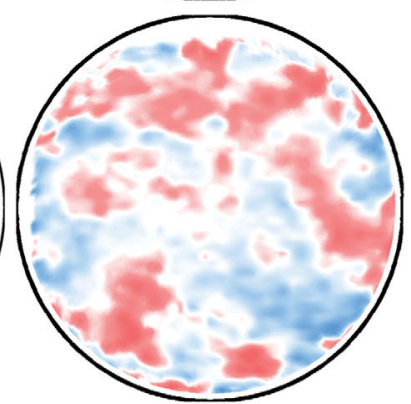

(b) Instantaneous example

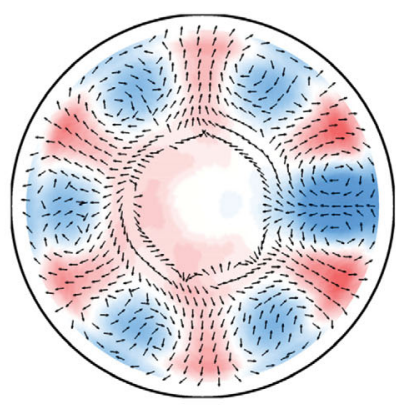

(c) Average for $u_{0}<0$

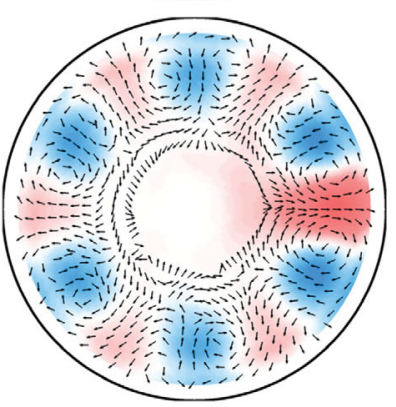

(d) Average for $u_{0}>0$

FIG. 1 (color). Organizational states at $\mathrm{Re}=35000$ corresponding to $k_{\theta}=2,3,4,5$, and 6 (top to bottom), shown by (a) the spatial correlation of the streamwise velocity fluctuation in the radial-azimuthal plane, red contours show $R_{u и}=0.05$ and 0.1 , blue contours $R_{u u}=-0.05$ and -0.1 ; the dot marks the center of the correlation at $\left(r_{0}, \theta_{0}\right)$, (b) instantaneous snapshots of the velocity field, (c) conditionally averaged velocity fields with $u_{0}<0$, (d) conditionally averaged velocity fields with $u_{0}>0$. In (b) - (d) the color maps show streamwise velocity fluctuations (red indicates $u>0$, blue indicates $u<0$, and white indicates $u \approx 0$ ) and vector arrows show the direction of the velocity in the azimuthal-radial plane. 
TABLE I. Properties of the five identified states.

\begin{tabular}{lccccc}
\hline \hline Wave number state & $k_{\theta}=2$ & $k_{\theta}=3$ & $k_{\theta}=4$ & $k_{\theta}=5$ & $k_{\theta}=6$ \\
\hline Vector fields assigned & $17 \%$ & $29 \%$ & $25 \%$ & $16 \%$ & $9 \%$ \\
Propensity to remain & $82 \%$ & $86 \%$ & $85 \%$ & $84 \%$ & $75 \%$ \\
Longest example & $1.2 R$ & $4.6 R$ & $2.0 R$ & $1.6 R$ & $1.8 R$ \\
\hline \hline
\end{tabular}

the $k_{\theta}=2$ state does resemble the edge state. This indicates that the fully turbulent flow at $\mathrm{Re}=35000$ still momentarily visits this state (or something similar) periodically and is a new insight into the behavior of turbulence at this relatively high Reynolds number. Each of the states we have identified $\left(k_{\theta}=2,3,4,5\right.$ and 6$)$ can be related to theoretical exact coherent states (traveling wave solutions) with $m$-fold rotational symmetry for $m=2,3,4,5$, and 6 found by Wedin and Kerswell at a Reynolds number an order of magnitude lower than our experiments [5].

The percentage of the vector fields assigned to each of the wave number states is shown in Table I. It is seen that the most common state is that of $k_{\theta}=3$ with $29 \%$. This corresponds to the very weak pattern visible in the total spatial correlation $[12,30]$ and the most dominant azimuthal mode found using proper orthogonal decomposition (POD) of simulated turbulent pipe flow [12]. It is possible that this is the fingerprint of the most common traveling wave solution in fully developed turbulent pipe flow at this Re. The state corresponding to $k_{\theta}=4$ is almost as common as $k_{\theta}=3$, being assigned to $25 \%$ of the vector fields investigated. This corresponds less well to POD results but it is very similar to another previously identified traveling-wave solution [4].

The experimental database allows the construction of a Markov chain that is representative of the behavior of turbulent pipe flow (solely in terms of the wave number states). Some properties of this chain are also included in Table I. The "propensity to remain" in the current state indicates the likelihood of the vector field $n+1$ being in the same azimuthal wave number state as vector field $n$. Vector fields are captured at the rate of 500 per second, so the time between field $n$ and $n+1$ being captured is $2 \mathrm{~ms}$. This propensity to remain is high for all wave number states, indicating that they have significant longevity. This longevity can be viewed as a streamwise extent by invoking Taylor's approximation of frozen flow [31,32]. As an indication of the potential longevity, the length of the "longest example" of each state (calculated using Taylor's approximation with a convection velocity equal to the bulk velocity) is also given in Table I. These values can be viewed as a measure of the stability of the state: the propensity to remain being as $\Delta t \rightarrow 0$ and the longest example being as $\Delta t \rightarrow \infty$. Together they indicate that all states are reasonably stable, but state $k_{\theta}=6$ is significantly less stable than the other four states that have been identified.
Figure 2 shows an example of the variation of the wave number states in the streamwise direction (top panel) through the use of Taylor's hypothesis. The axial coherence of each of the states is demonstrated by this figure. In this particular case $k_{\theta}=3$ shows very strong coherence, but this is just one example. The middle panel is the corresponding correlation $\left(R_{u u}\right)$ for each streamwise location. This clearly shows several examples of transitions between states (e.g., at $x / R \approx 2,6,10,16,20$ ). The bottom panel is the corresponding instantaneous streamwise velocity fluctuation, which enables the visualization of the large-scale structures that are responsible for the pattern in $R_{u u}$ shown in the middle panel, and are therefore key in determining the wave number state of the flow.

Figure 1 of this Letter perfectly encapsulates the first evidence that exact coherent states (i.e., unstable nonlinear traveling-wave solutions to the Navier-Stokes equations that one might previously have considered to be phenomena solely of transition) have a significant imprint on turbulent pipe flow at a Reynolds number of $\mathrm{Re}=35000$, which is an order of magnitude above transition. Importantly, these are the first results at any Reynolds number to show the presence of these solutions
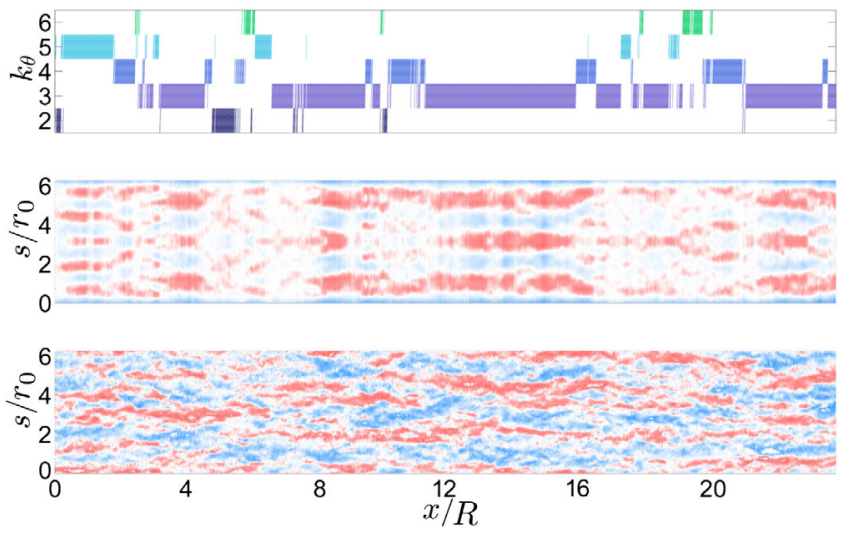

FIG. 2 (color online). An example of the axial coherence of the wave number states. Top: Variation of azimuthal wave number $\left(k_{\theta}\right)$ with axial distance $(x)$, showing significant axial coherence of the wave number states. Middle: The corresponding correlation map at $r_{0} / R=0.75$ showing the correlations that lead to the state allocation and also the transitions between wave number states (red indicates $R_{u и}>0$, blue $R_{u и}<0$, white $R_{u и} \approx 0$ and $s$ is the arclength, i.e., $s=r_{0} \theta$ ). Bottom: The corresponding instantaneous velocity fluctuations, where red indicates $u>0$, blue $u<0$, and white $u \approx 0$. 
statistically and not just through instantaneous snapshots. This is crucial in our cognizance of the importance of these states and the potential they have to enhance our fundamental understanding of turbulence at any Reynolds number and potentially allow us to control it.

*David.Dennis@liverpool.ac.uk

[1] G. Kawahara, M. Uhlmann, and L. van Veen, Annu. Rev. Fluid Mech. 44, 203 (2012).

[2] F. Waleffe, Phys. Rev. Lett. 81, 4140 (1998).

[3] F. Waleffe, J. Fluid Mech. 435, 93 (2001).

[4] H. Faisst and B. Eckhardt, Phys. Rev. Lett. 91, 224502 (2003).

[5] H. Wedin and R. R. Kerswell, J. Fluid Mech. 508, 333 (2004).

[6] C. C. T. Pringle and R. R. Kerswell, Phys. Rev. Lett. 99, 074502 (2007).

[7] A. P. Willis and R. R. Kerswell, Phys. Rev. Lett. 100, 124501 (2008).

[8] A. de Lozar, F. Mellibovsky, M. Avila, and B. Hof, Phys. Rev. Lett. 108, 214502 (2012).

[9] M. Avila, F. Mellibovsky, N. Roland, and B. Hof, Phys. Rev. Lett. 110, 224502 (2013).

[10] J. Westerweel, G. E. Elsinga, and R. J. Adrian, Annu. Rev. Fluid Mech. 45, 409 (2013).

[11] P. Moin and K. Mahesh, Annu. Rev. Fluid Mech. 30, 539 (1998).

[12] J. R. Baltzer, R. J. Adrian, and X. Wu, J. Fluid Mech. 720, 236 (2013).

[13] R. J. Adrian, C. D. Meinhart, and C. D. Tomkins, J. Fluid Mech. 422, 1 (2000).

[14] B. Ganapathisubramani, E. K. Longmire, and I. Marusic, J. Fluid Mech. 478, 35 (2003).
[15] B. Ganapathisubramani, N. Hutchins, W. T. Hambleton, E. K. Longmire, and I. Marusic, J. Fluid Mech. 524, 57 (2005).

[16] J. C. del Alamo, J. Jimenez, P. Zandonade, and R. D. Moser, J. Fluid Mech. 561, 329 (2006).

[17] R. J. Adrian, Phys. Fluids 19, 041301 (2007).

[18] D. J. C. Dennis and T. B. Nickels, J. Fluid Mech. 673, 218 (2011).

[19] G. E. Elsinga and J. Westerweel, Exp. Fluids 52, 865 (2012).

[20] B. J. Balakumar and R. J. Adrian, Phil. Trans. R. Soc. A 365, 665 (2007).

[21] N. Hutchins and I. Marusic, J. Fluid Mech. 579, 1 (2007).

[22] J. P. Monty, J. A. Stewart, R. C. Williams, and M. S. Chong, J. Fluid Mech. 589, 147 (2007).

[23] D. J. C. Dennis and T. B. Nickels, J. Fluid Mech. 673, 180 (2011).

[24] J. Lee and H. Sung, Phys. Fluids 25, 045103 (2013).

[25] C. W. H. van Doorne and J. Westerweel, Exp. Fluids 42, 259 (2007).

[26] T. M. Schneider, B. Eckhardt, and J. Vollmer, Phys. Rev. E 75, 066313 (2007).

[27] B. Hof, C. W. H. van Doorne, J. Westerweel, F. T. M. Nieuwstadt, H. Faisst, B. Eckhardt, H. Wedin, R. R. Kerswell, and F. Waleffe, Science 305, 1594 (2004).

[28] B. Hof, C. W. H. van Doorne, J. Westerweel, and F. T. M. Nieuwstadt, Phys. Rev. Lett. 95, 214502 (2005).

[29] T. M. Schneider, B. Eckhardt, and J. A. Yorke, Phys. Rev. Lett. 99, 034502 (2007).

[30] S. C. C. Bailey, M. Hultmark, A. J. Smits, and M. P. Schultz, J. Fluid Mech. 615, 121 (2008).

[31] G. I. Taylor, Proc. R. Soc. A 164, 476 (1938).

[32] D. J. C. Dennis and T. B. Nickels, J. Fluid Mech. 614, 197 (2008). 